\title{
Quadrupolar Ordered State at High Fields in $\mathrm{PrPb}_{3}$
}

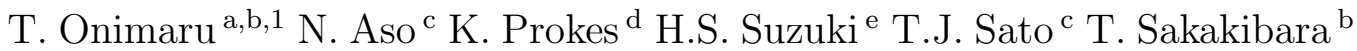 \\ a SORST, Japan Science and Technology Agency, Kawaguchi, Saitama 332-0012, Japan \\ ${ }^{\mathrm{b}}$ Institute for Solid State Physics, University of Tokyo, Kashiwa, Chiba 277-8581, Japan

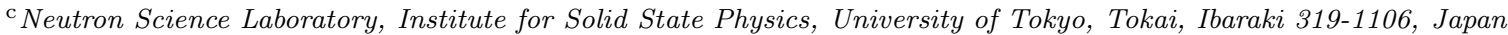 \\ ${ }^{\mathrm{d}}$ Hahn-Meitner-Institut, SF-2, Berlin, Germany \\ e National Institute for Materials Science, Tsukuba 305-004\%, Japan
}

\begin{abstract}
$\mathrm{PrPb}_{3}$ exhibits an antiferroquadrupolar transition at $T_{\mathrm{Q}}=0.4 \mathrm{~K}$. In the present work, in order to reveal the order parameters of the ordered states in much higher fields than $5 \mathrm{~T}$ applied along the [11̄0] direction, we performed neutron diffraction experiments in the temperature range between $50 \mathrm{mK}$ and $0.8 \mathrm{~K}$ in magnetic fields up to $14.5 \mathrm{~T}$. Above 7

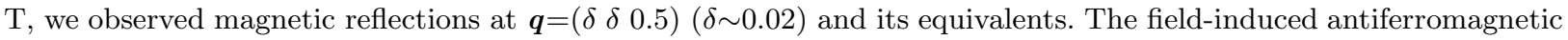
components were normal to the applied field, indicating that the quadrupolar order parameter in the high field phase should be of $\mathrm{O}_{2}^{2}$-type.
\end{abstract}

Key words: Antiferroquadrupolar ordering, Neutron diffraction PACS: $71.20 . \mathrm{Eh} ; 75.20 . \mathrm{Hr} ; 75.50 . \mathrm{Kj}$

\section{Introduction}

Antiferroquadrupolar (AFQ) ordering in the intermetallic $f$ electron compounds has attracted much interest in these years. $\mathrm{PrPb}_{3}$ crystallizes in a simple $\mathrm{AuCu}_{3}$-type cubic structure, and has a $\Gamma_{3}$ non-Kramers doublet in the crystalline-electricalfield $(\mathrm{CEF})$ ground state, with a magnetic $\Gamma_{4}$ triplet lying $15 \sim 19 \mathrm{~K}$ above the ground state.[1,2] Since the $\Gamma_{3}$ doublet carries quadrupole moments such as $O_{2}^{0}$ and $O_{2}^{2}, \mathrm{PrPb}_{3}$ is a good candidate for a quadrupolar transition. Actually, at the temperature of $T_{\mathrm{Q}}=0.4 \mathrm{~K}$, a second order transition was observed with a lambda type anomaly in the spe-

\footnotetext{
* Corresponding author. Tel.: +81-471363298; fax: +81471346069 .

E-mail address: onimaru@issp.u-tokyo.ac.jp (T. Onimaru). 1 Present address; Department of Quantum Matter, ADSM, Hiroshima University, 1-3-1 Kagamiyama, HigashiHiroshima, Hiroshima 739-8530, Japan
}

cific heat measurement.[3,4] The idea of an AFQ ordering has further been strengthened by the $H-T$ diagram study[5], in which reentrant behavior with a significant enhancement of the transition temperature $T_{\mathrm{Q}}(H)$ was observed for $H \|\langle 100\rangle$, as is often the case for AFQ ordering systems. Moreover, angle-resolved magnetization measurements revealed characteristic field-angular oscillations of $T_{\mathrm{Q}}(H)$, which can be interpreted by assuming an $\mathrm{O}_{2}^{0}$-type AFQ moment and its equivalents to be the order parameters (OPs) at low $H(<6 \mathrm{~T}) .[6,7]$

Very recently, we have revealed the quadrupolar structure in the AFQ phase by detecting an antiferromagnetic component induced by magnetic field applied along the [001] direction.[8] Interestingly, the quadrupoles are distributed with the sinusoidally modulated structure, suggesting that the RKKY-type interaction between the quadrupoles plays an important role in this system. $[9,10]$ Moreover, the direction of the field-induced magnetic 
moments is parallel to the magnetic field, indicating the quadrupolar OP in the AFQ phase should be of $O_{2}^{0}$-type.[11] In much higher magnetic fields than $1 \mathrm{~T}$, the sinusoidal structure turns into an antiphase structure with decreasing temperature below $T_{\mathrm{t}}\left(<T_{\mathrm{Q}}\right)$. On the other hand, when magnetic field was applied along the $[01 \overline{1}]$ direction, no superlattice reflection was observed on the $(h k k)$ scattering plane $(\perp \boldsymbol{H})$ below $3 \mathrm{~T}$, although weak reflections at $\boldsymbol{q}=(0.5 \delta \delta)(\delta \sim 0.062)$ and its equivalents were observed only in the fields of $3 \mathrm{~T}<H<5 \mathrm{~T}$ at $80 \mathrm{mK}$.[12] From the analysis of the $\boldsymbol{Q}$-dependence of the reflection intensity, the field-induced component is determined to be perpendicular to the field, suggesting that the $\mathrm{OP}$ should be of $\mathrm{O}_{2}^{2}$-type.[11] The $\mathrm{O}_{2}^{2}$-type OP was also suggested from the measurements of the field angle dependence of the transition temperature; a new phase characterized by the transition temperature $T_{\mathrm{Q}}$ ' was observed in fields above $7 \mathrm{~T}$ in narrow field directions around the $\langle 110\rangle$ axis. [7] However, the ordered state in the magnetic fields above $5 \mathrm{~T}$ has not been revealed by a microscopic techniques yet.

In the present work, in order to reveal the OPs of the ordered states in much higher fields than $5 \mathrm{~T}$ applied along the [1 $\overline{1} 0]$ direction, we performed neutron diffraction experiment at temperatures ranging from $50 \mathrm{mK}$ to $0.8 \mathrm{~K}$ and in magnetic fields up to $14.5 \mathrm{~T}$. Above $7 \mathrm{~T}$, we have found field-induced magnetic reflections at $\boldsymbol{q}=\left(\begin{array}{lll}\delta & \delta & 0.5\end{array}\right)(\delta \sim 0.02)$ and its equivalents. The $\boldsymbol{Q}$-dependence of their intensity indicates that the field induced antiferromagnetic component is perpendicular to the field, confirming that the quadrupolar OP of this new ordered phase should be of $O_{2}^{2}$-type. In the fields of $3 \mathrm{~T} \leq H \leq 7 \mathrm{~T}$, we have also observed some reflections, for example,

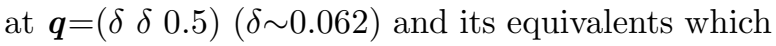
were reported before.[12]

\section{Results and Discussion}

A single crystalline sample of $\mathrm{PrPb}_{3}$ was grown by the Bridgman method using a Mo crucible. In the present study, a specimen with dimensions of $10 \times 8 \times 5 \mathrm{~mm}^{3}$ mounted on a mixing chamber of a ${ }^{3} \mathrm{He}-{ }^{4} \mathrm{He}$ dilution refrigerator (base temperature 50 $\mathrm{mK}$ ) was placed in a vertical superconducting cryomagnet capable to generate $14.5 \mathrm{~T}$. The magnetic field was applied along the $[1 \overline{1} 0]$ direction, therefore, the scattering plane was the $(h h l)$ plane perpendicular to the magnetic field. Neutron diffraction measurements were performed using the double-axis spectrometer E4 installed at the HMI with incident neutron wave length of $2.44 \AA$.

Figure 1 shows the $\boldsymbol{Q}$-scan along the $(h h 0.5)$ line at the temperature of $T=0.1 \mathrm{~K}$ and in various magnetic fields of $0 \leq H \leq 14.5 \mathrm{~T}$. Below $3 \mathrm{~T}$, we could not observe any magnetic reflections neither on the $(h$ $h 0.5)$ line nor along the principal lines on the $(h h l)$ plane such as the $(h h 0),(00 l)$ and $(h h l)$ lines. Increasing the magnetic field above $7 \mathrm{~T}$, magnetic reflections appeared at $\boldsymbol{q}=( \pm \delta \pm \delta 0.5)(\delta \sim 0.02)$ and their equivalents. The intensity increases with increasing $H$ from $7 \mathrm{~T}$ to $10 \mathrm{~T}$, but turns to decrease at higher fields. Even at the maximum field of $14.5 \mathrm{~T}$, however, the reflections still remain at $0.1 \mathrm{~K}$. We also observed their third harmonic reflections at around $10 \mathrm{~T}$. They probably result from squaring-up of a modulated structure as will be shown later. We observed the small superlattice reflections at $\boldsymbol{q}=(\delta \delta$ $0.5)(\delta \sim 0.062)$ and their equivalents in the fields of 3 $\mathrm{T} \leq H \leq 7 \mathrm{~T}$. This result confirmed our previous data obtained at $T=40 \mathrm{mK}$ and in the magnetic fields of $3 \mathrm{~T} \leq H \leq 5 \mathrm{~T}$.[12] Moreover, in the field of $6 \mathrm{~T}<H<7$ $\mathrm{T}$, we observed other superlattice reflections in the ( $h$ h 0.5 ) line. Although we are not able to determine the structures from the reflections because they are very small and undefined, they probably result from instability of the OPs shown as follow.

In order to confirm the phase boundary between the AFQ ordered and the paramagnetic states, we examined the temperature dependence of the intensity of the magnetic reflections. Figure 2 shows the evolution of the integrated intensity of the magnetic reflection at $\boldsymbol{q}=(-\delta-\delta 0.5)(\delta \sim 0.02)$ in the magnetic field of $H=10 \mathrm{~T}$. The dotted line shows the background of the intensity. The intensity decreases continuously with increasing $T$, and it diminished to the back ground level at the transition temperature of $T_{\mathrm{Q}}^{\prime}=0.58 \mathrm{~K}$ as shown with the thick arrow in Fig. 2, indicating that the ordered state collapses above $T_{\mathrm{Q}}^{\prime}$. We also examined the temperature dependence of the intensity in various fields. The transition temperature $T_{\mathrm{Q}}^{\prime}$ varies with changing the magnetic fields. From the results, we confirmed that the field dependence of $T_{\mathrm{Q}}^{\prime}$ thus obtained was in good agreement with the $H-T$ phase diagram determined by our magnetization measurements. [7] On the other hand, the boundary of the ordered phase characterized by the appearance of the magnetic reflection at $\boldsymbol{q}=(\delta \delta 0.5)(\delta \sim 0.062)$ could not be clearly defined because of the weakness of the intensity. 
The magnetic structure in the ordered phases can be derived from the $\boldsymbol{Q}$-dependence of the intensity of the magnetic reflections. Figure 3 shows the antiferromagnetic components of the field-induced magnetic structure determined from the magnetic reflections at $\boldsymbol{q}=( \pm \delta \pm \delta 0.5)(\delta \sim 0.02)$ and their equivalents at $T=0.1 \mathrm{~K}$ and in the magnetic field of $H=10$ $\mathrm{T}$. We found that the intensity of the reflection at

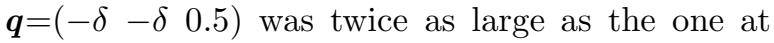
$\boldsymbol{q}=\left(\begin{array}{lll}\delta & \delta & 0.5\end{array}\right)$. In the ideal condition, the intensity of these two peaks should coincide with each other. The disparity could possibly be attributed to a misalignment of the field direction. We therefore took the average of these satellite intensity for analysis of the magnetic structure. We assumed that antiferromagnetic component is parallel to the [110] direction and is modulated with the propagation vector of $\boldsymbol{k}=[ \pm \delta \pm \delta 0.5]$ ( $\delta \sim 0.02)$. Fig. 3 shows the alignment of the moment in the $z=0$ plane. The direction of each moment changes alternatively along the [001] direction. We determined the amplitude of the sinusoidal structure to be $0.93(15) \mu_{\mathrm{B}}$. The fact that the antiferromagnetic component is perpendicular to the field suggests that the quadrupolar OP in the ordered phase should be the $O_{2}^{2}$-type.[11] The uniform component of the magnetic moments at $10 \mathrm{~T}$ estimated from the field-enhanced intensity of the nuclear Bragg peaks was 1.52(20) $\mu_{\mathrm{B}}$, being consistent with the results of the magnetization measurements.[7]

We now discuss the possible OPs in the ordered phases. In the magnetic field applied along the $\langle 100\rangle$ direction, the OP has been confirmed to be of $\mathrm{O}_{2}^{0}-$ type by our previous neutron diffraction experiment in magnetic fields, where the antiferromagnetic component of the field-induced magnetic moments is parallel to the field. [8] This is consist with the OP suggested by the angular dependence of the transition temperature $T_{\mathrm{Q}}$. [6] The quadrupolar ordering temperature $T_{\mathrm{Q}}$ varies with rotating the field. $T_{\mathrm{Q}}$ has a maximum value in the $\langle 100\rangle$ direction and a cusp-type minimum in the $\langle 110\rangle$ direction below 4 T. These results were well explained by assuming that the low field phase consists of $\mathrm{O}_{2}^{0}$-type OP with three domains as explained in detail in Ref. [6]. On the other hand, in the angle-resolved magnetization measurements performed at higher fields, $T_{\mathrm{Q}}$ ' appeared in the fields above $7 \mathrm{~T}$ in a narrow angular range very close to the $\langle 110\rangle$ axis. [7] Considering that the $\Gamma_{3}$ doublet carries the quadrupoles $O_{2}^{0}$ and $\mathrm{O}_{2}^{2}$, the $\mathrm{OP}$ of the ordered phase observed around the $\langle 110\rangle$ direction above $7 \mathrm{~T}$ was expected to be of $O_{2}^{2}$-type. This point has strongly been supported by the present neutron experiment in which the antiferromagnetic component of the $\langle 110\rangle$ high field phase is perpendicular to the field, being compatible with the $O_{2}^{2}$ AFQ OP.[11] The present neutron data, combined with our magnetization and neutron experiments, indicates that the OP changes from $O_{2}^{0}$ to $O_{2}^{2}$ types in magnetic fields along the $\langle 110\rangle$ direction. This change of the OPs in magnetic fields would probably result from the anisotropic interaction between the quadrupoles, where the $O_{2}^{0}$ type OP is preferred over the $O_{2}^{2}$ one in zero field. This was also pointed out by the angle-resolved magnetization measurements.[6] The complicated and strongly anisotropic phase diagrams of $\mathrm{PrPb}_{3}$ strongly suggests the relevance of the anisotropic quadrupolar interaction in this system.

\section{Conclusion}

In summary, we performed the neutron diffraction experiments on $\mathrm{PrPb}_{3}$ in the magnetic fields up to $14.5 \mathrm{~T}$ applied along the $\langle 110\rangle$ direction. Above 7 $\mathrm{T}$, the magnetic reflections were observed at $\boldsymbol{q}=( \pm \delta$ $\pm \delta 0.5)(\delta \sim 0.02)$ and its equivalents. In the field of $3 \mathrm{~T}<H<7 \mathrm{~T}$, the weak reflections were observed, for example, at $\boldsymbol{q}=( \pm \delta \pm \delta 0.5)(\delta \sim 0.062)$ and its equivalents. On the other hand, no superlattice reflection was observed below $3 \mathrm{~T}$ in the $(h h l)$ scattering plane. The quadrupolar $\mathrm{OP}$ in the ordered phase above 7 $\mathrm{T}$, characterized by the transition temperature $T_{\mathrm{Q}}$, was confirmed to be of $\mathrm{O}_{2}^{2}$-type, which is consistent with the results of the angle-resolved magnetization measurements. The results suggest that the OP should change from the $O_{2}^{0}$-type to the $O_{2}^{2}$-type by applying the magnetic field along the $\langle 110\rangle$ direction. This behavior of the OPs and the anisotropic and complex phase diagrams probably result from the anisotropic RKKY-type interaction between the quadrupoles.

\section{Acknowledgment}

We thank T. Kawae, R. Watanuki, T. Morie, S. Jumonji, Y. Nemoto and T. Goto for helpful discussions. The present work was supported by Grantsin-Aid for Scientific Research from JSPS and the MEXT of Japan and SORST, Japan Science and Technology Agency. 


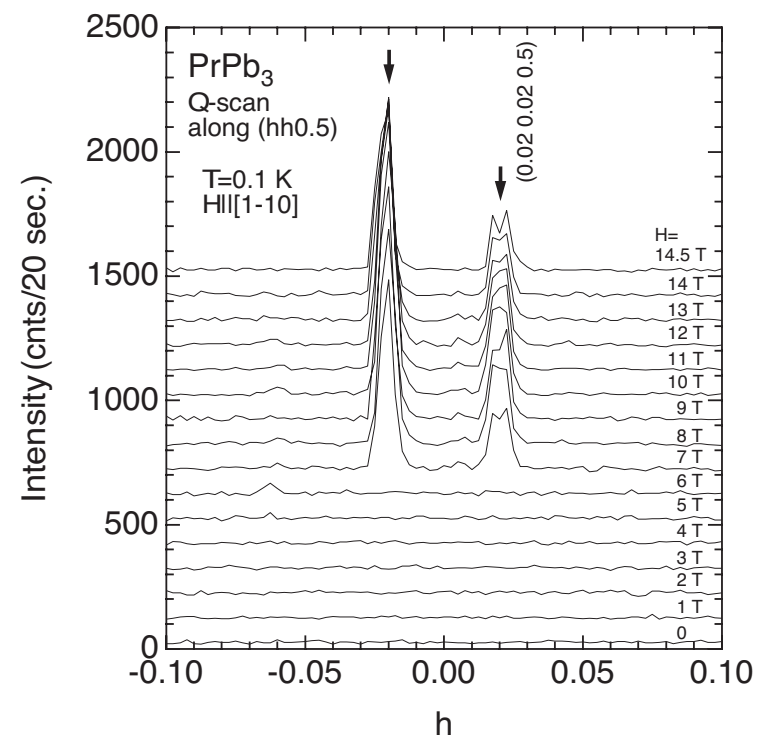

Fig. 1. $Q$-scan along the ( $h h 0.5)$-line at the temperature of $T=0.1 \mathrm{~K}$ in the magnetic fields up to $14.5 \mathrm{~T}$ applied along the $[1 \overline{1} 0]$ direction. Each curve is vertically shifted for clarity.

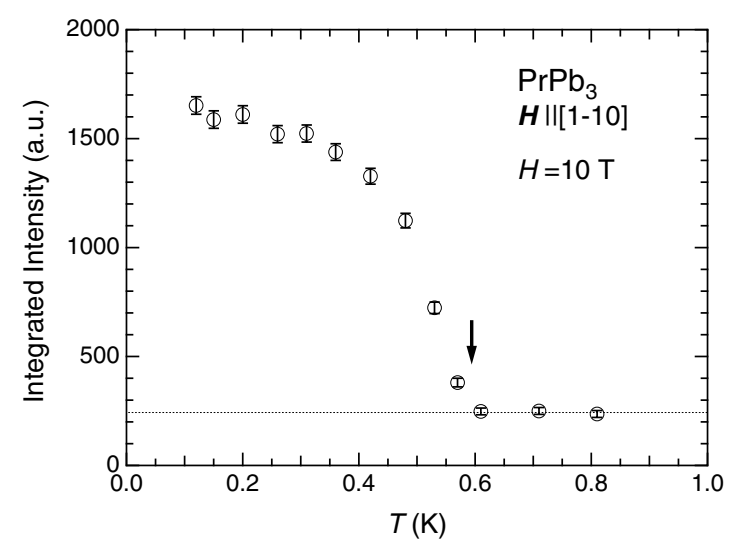

Fig. 2. Temperature dependence of the integrated intensity of the field-induced magnetic reflection at $\boldsymbol{q}=(\delta \delta 0.5)(\delta \sim 0.02)$ in the magnetic field of $H=10 \mathrm{~T}$ applied along the [110] direction. The dotted line shows the background of the intensity. The thick arrow indicates the transition temperature $T_{\mathrm{Q}}^{\prime}$.

\section{References}

[1] W. Gross, K. Knorr, A.P. Murani and K.H.J. Buschow, Z. Phys. B 37 (1980) 123.

[2] M. Niksch, W. Assmus, B. Lüthi, H.R. Ott and J.K. Kjems, Helv. Phys. Acta 55 (1982) 688.

[3] E. Bucher, K. Andres, A.C. Gossard and J.P. Maita, J. Low Temp. 2 (1972) 322.

[4] D. Aoki et al., J. Phys. Soc. Jpn. 66 (1997) 3988.

[5] T. Tayama et al., J. Phys. Soc. Jpn. 70 (2001) 248.

[6] T. Onimaru et al., J. Phys. Soc. Jpn. 73 (2004) 2377.



Fig. 3. Field-induced magnetic structure determined by the $Q$ dependence of the intensity of the magnetic reflection at $\boldsymbol{q}=( \pm \delta \pm \delta \quad 0.5) \quad(\delta \sim 0.02)$ and their equivalents at the temperature of $T=0.1 \mathrm{~K}$ and in the magnetic field of $H=10$ $\mathrm{T}$. The antiferromagnetic component is parallel to the [110] direction and the components are modulated with the amplitude of $\mu_{\mathrm{AF}}=0.93(15) \mu_{\mathrm{B}}$ and the propagation vector of $\boldsymbol{k}=\left[\begin{array}{lll} \pm \delta & \pm \delta & 0.5\end{array}\right] \quad(\delta \sim 0.02)$. The magnetic moments are distributed alternatively along the [001] direction.

[7] T. Onimaru et al., in preparation.

[8] T. Onimaru et al., Phys. Rev. Lett. 94 (2005) 197201.

[9] D. Schmitt and P. M. Levy: J. Mag. Mag. Mater. 49 (1985) 15 .

[10] P.M. Levy, P. Morin and D. Schmitt, Phys. Rev. Lett. 42 (1979) 1417.

[11] R. Shiina, H. Shiba and P. Thalmeier: J. Phys. Soc. Jpn. 66 (1997) 1741.

[12] T. Onimaru et al., Physica B 359-361 (2005) 935. 